\title{
SOLUTION OF THE TRANSIENT THERMAL DIFFUSION EQUATION USING THE TIME-DEPENDENT BOUNDARY ELEMENT METHOD
}

\author{
K.E. DONNE, A. MAROTIN \& T. BASHFORD \\ Faculty of Architecture, Computing \& Engineering, University of Wales Trinity Saint David, Swansea, UK.
}

\begin{abstract}
A time-dependent fully-parallelised formulation of the BEM is applied to transient thermal problems in the context of light-based medical devices. The method is initially verified against benchmark problems. The limitations of the model are discussed, particularly the singularity challenge inherent in the fundamental solution. The method is then applied for a representative 3D clinical problem, further illustrating the singularity challenge.

Keywords: boundary element, diffusion, parallel, thermal.
\end{abstract}

\section{INTRODUCTION}

The problem being considered is the application of pulsed, collimated light incident onto human tissue, targeting structures such as hair follicles, tumours or blood vessels. The computation usually consists of two stages: Stage 1, where the radiative transport equation is solved for incident low-energy photons. This calculation generates a volumetric distribution of light fluence throughout the tissue, which in turn provides the time-varying heat source terms for Stage 2, the time-dependent thermal calculation. This generates the time-evolving temperature distribution throughout the tissue volume, facilitating estimates of thermal damage to the desired sub-surface target to be made for clinical planning. In the current work, the radiative transport equation is solved using the Dual Reciprocity Boundary Element Method (DRBEM) and the time-dependent thermal calculation is solved using the Time-Dependent Boundary Element Method (TDBEM). We have previously reported [1] on our application of the DRBEM. This paper concentrates on the applicability of the TDBEM, with benchmarking for simple geometries establishing the method's accuracy and a subsequent case study of a three-dimensional tissue problem where the sub-surface structure - a hair follicle - is represented by an unstructured mesh.

\section{THE TIME-DEPENDENT BEM FORMULATION}

We consider the integral form of the thermal diffusion equation:

$$
\nabla^{2} T(x, y, z, t)-\frac{1}{\alpha} \frac{\partial T(x, y, z, t)}{\partial t}+\frac{b(x, y, z, y)}{k}=0
$$

where $t$ is time, $T$ is the temperature, $b(x, y, z, t)$ is a heat source term, $\alpha=k /(\rho c)$ is the thermal diffusivity $\left[\mathrm{m}^{2} \cdot \mathrm{s}^{-1}\right], k$ is the thermal conductivity $\left[\mathrm{W} \cdot \mathrm{m}^{-1} \cdot \mathrm{K}^{-1}\right], \rho$ the density $\left[\mathrm{kg} \cdot \mathrm{m}^{-3}\right]$, and $c$ the specific heat $\left[\mathrm{J} \cdot \mathrm{kg}^{-1} \cdot \mathrm{K}^{-1}\right]$.

The fundamental solution is of the form

$$
T^{*}\left(s, p, t_{F}, t\right)=\frac{1}{(4 \pi \alpha t)^{d / 2}} \exp \left(\frac{-r^{2}}{4 \alpha \tau}\right) H(\tau)
$$


and describes the influence of a source point at a time $t$ and location $s$ on a field point at location $p$ and time $t_{F}$, where $\tau=t_{F}-t ; r$ is the distance between the source point $s$ and the field point $p$, and $d$ is the number of spatial dimensions of the problem. See Wrobel [2] for details.

The general form of the integral equation equivalent to eqn (1), valid for any position of $s$ considered (either in the domain $\Omega$ or on the boundary $\Gamma$ ) is

$$
\begin{gathered}
C(s) T\left(s, t_{F}\right)+a \int_{t_{0}}^{t_{F}} \int_{\Gamma} T(p, t) q^{*}\left(s, p, t_{F}, t\right) d \Gamma d t \\
=\alpha \int_{t_{0}}^{t_{F}} \int_{\Gamma} q(p, t) T^{*}\left(s, p, t_{F}, t\right) d \Gamma d t+\int_{\Omega} T_{0}(p) T^{*}\left(s, p, t_{F}, t_{0}\right) d \Omega
\end{gathered}
$$

Where $C(s)$ is a constant; $T_{0}$ is the initial temperature distribution over the domain and $q^{*}$ is the normal derivative of $T^{*}$.

The internal heat generation term due to the absorbed photon flux (obtained from a previous DRBEM calculation) is given by the following domain integral over space and time

$$
I_{b}\left(s, t_{F}\right)=a \int_{t_{0}}^{t_{F}} \int_{\Omega} b(p, t) T^{*}\left(s, p, t_{F}, t\right) d \Omega d t
$$

which is added to the RHS in eqn (3).

\subsection{Time-integration schemes}

Brebbia et al. [3] present two different time-marching schemes that can be employed in the numerical solution of eqn (3).

The main difference between the two time-marching schemes possible in the numerical solution of eqn (3) lies in the way in which the values of temperature and flux, up to the current time, are taken into account when solving for a new time step. In scheme 1, these values are accounted for through a domain integral, as pseudo-initial values, with the time integration only over the range $t_{F-1}$ to $t_{F}$. The attraction of scheme 1 is that, for a constant time step, the Green's matrices can be computed once and stored, although the scheme involves a domain integration. In scheme 2, the temperature at a future time step is considered through a summation of boundary integrals over the entire time from $t_{0}$ to $t_{F}$. The Green's matrices are then more cumbersome and the computational overheads become unreasonable for a large number of time steps. We therefore concentrate on Scheme 1.

Scheme 1: each time step is considered as a new problem and so, at the end of each step, values of the temperature field have to be computed at a sufficient number, $L$, of domain points in order to use them as pseudo-initial values for the next step. This approach minimising the time integrations is very efficient except for the requisite domain integration in eqn (3) associated with the temperature field $T_{F-1}$ at each step.

The initial conditions are then taken into account through a numerical integration over the domain and their values at the $L$ internal poles.

It is assumed that the boundary $\Gamma$ and the domain $\Omega$ are discretized into $N$ constant boundary elements and $L$ domain cells respectively, and the time dimension is subdivided into $F$ time steps. Thus, for a point $i$ on the boundary, the following discretized equations is obtained 


$$
C_{i} T_{i, F}+\alpha \sum_{j=1}^{N} \int_{\Gamma_{j}}^{t_{f}} \int_{t_{F-1}} T q^{*} d t d=\alpha \sum_{j=1}^{N} \int_{\Gamma_{j}}^{t_{f}} \int_{t_{F-1}} q T^{*} d t d \Gamma+\sum_{l=1}^{L} \int_{\Omega_{l}} T^{*} T_{F-1} d \Omega
$$

Assuming that functions $T$ and $q$ do not vary in time over each time step, applying eqn (5) to all boundary nodes yields the following system of equations

$$
\mathrm{HT}_{\mathrm{F}}=\mathrm{G} \mathrm{Q}_{\mathrm{F}}+\mathrm{B} \overline{\mathrm{T}}_{\mathrm{F}-1}
$$

where

$$
\begin{gathered}
H_{i j}=\alpha \int_{\Gamma_{j}} \int_{t_{F-1}}^{t_{F}} q^{*}\left(i, j, t_{F}, t\right) d t d \Gamma_{j} \\
G_{i j}=a \int_{\Gamma_{j}}^{t_{f}} \int_{t_{F-1}} T^{*}\left(i, j, t_{F}, t\right) d t d \Gamma_{j} \\
B_{i l}=\int_{\Omega} T^{*}\left(i, l, t_{F}, t_{F-1}\right) d \Omega_{l}
\end{gathered}
$$

Details of the integration of eqns (7)-(9) are well documented in, for example, Wrobel [2].

The temperature throughout the domain is known at time $t_{0}$. If half the boundary values of $T$ and $q$ are prescribed, eqn (6) can then be used to compute the remaining boundary data for the first time step $(F=1)$.

At the end of a time step, the values of $T$ at the $L$ internal poles are recomputed to be used as initial values for the next step. This can be done by using the following equation

$$
\overline{\mathbf{T}}_{\mathrm{F}}=\mathbf{G}^{\prime} \mathbf{Q}_{\mathrm{F}}-\mathbf{H}^{\prime} \mathbf{T}_{\mathrm{F}}+\mathbf{B}^{\prime} \overline{\mathbf{T}}_{\mathrm{F}-1}
$$

The dimensions of the different matrices in eqns (6) and (10) are:

- G: dimension $N \times N$

- H: dimension $N \times N$

- B: dimension $N$ x $L$
- $\mathbf{G}^{\prime}$ : $\operatorname{dimension} N \times L$

- $\mathbf{H}^{\prime}$ : dimension $N \times L$

- $\mathbf{B}^{\prime}$ : dimension $N_{v}$ x $L$

where $N_{v}$ is the number of domain points where the solution is required, as distinct from the $L$ internal poles used in the TDBEM algorithm.

The coefficients of these matrices depend on geometrical data, properties of the medium and the time step. Thus, adopting a constant time step throughout the analysis, they can all be computed only once and stored.

For problems with known initial temperatures throughout the domain and the boundary, the solution procedure is then:

1. Compute the coefficients of matrices $\mathbf{G}, \mathbf{H}, \mathbf{B}$ in eqn (6) and $\mathbf{G}^{\prime}, \mathbf{H}^{\prime}, \mathbf{B}^{\prime}$ in eqn (10) using the specified values of geometrical data, properties of the medium and time step. Compute the inverse of matrix $\mathbf{G}$. Introduce the boundary conditions and multiply them by matrices $\mathbf{H}$ and $\mathbf{H}^{\prime}$ to form the vectors of independent terms $\mathbf{F}(N)$ and $\mathbf{F}^{\prime}(L)$, respectively such as

$$
\begin{aligned}
& \mathbf{F}=\mathbf{H}_{i j} \mathbf{T}_{j, F} \quad i, j \in \Gamma \\
& \mathbf{F}^{\prime}=\mathbf{H}_{l j}^{\prime} \mathbf{T}_{j, F} \quad l \in \Omega, j \in \Gamma
\end{aligned}
$$


Store the four matrices $\mathbf{G}^{-1}, \mathbf{H}, \mathbf{G}^{\prime}, \mathbf{H}^{\prime}$ and the two vectors $\mathbf{F}$ and $\mathbf{F}$ '.

2. Multiply matrix $\mathbf{B}$ by the initial conditions, subtract from vector $\mathbf{F}$ and multiply the result by $\mathbf{G}^{-1}$ to find the unknown boundary values of $q$

$$
\mathbf{Q}_{j, F}=\mathbf{G}_{i j}^{-1}\left(\mathbf{F}-\mathbf{B}_{i l} \overline{\mathbf{T}_{l, F-1}}\right) \quad i, j \in \Gamma, \quad l \in \Omega
$$

3. Multiply matrix $\mathbf{B}^{\prime}$ by the initial conditions, subtract $\mathbf{F}^{\prime}$ and add the result of the product of $\mathbf{G}^{\prime}$ by the computed boundary values of $\mathbf{Q}$ to find the initial conditions for the next step

$$
\overline{\mathbf{T}_{l, F}}=\mathbf{G}_{l i} \mathbf{Q}_{i, F}-\mathbf{F}^{\prime}+\mathbf{B}{ }_{l k} \overline{\mathbf{T}_{k, F-1}} \quad l, k \in \Omega, i \in \Gamma
$$

4. Compare $\overline{\mathbf{T}_{l, F}}$ and $\overline{\mathbf{T}_{l, F-1}}$ to verify if a steady-state is reached, i.e. if their difference is less than a prescribed tolerance tol. If it is not the case return to step 3. and carry on until $\left|\overline{\mathbf{T}_{l, F}}-\overline{\mathbf{T}_{l, F-1}}\right| \leq$ tol .

\section{VERIFICATION OF NUMERICAL MODEL}

\subsection{Dirichlet boundary conditions and no volumetric heat source term}

We now consider a cube, as shown in Fig. 1, with zero initial temperature and prescribed surface temperature of $10^{\circ} \mathrm{C}$ as a constant boundary condition. The cube's boundary is discretized into 1,200 triangles and its volume into 3,669 tetrahedrons within which temperature and flux are assumed to be constant. Constant and linear time interpolation functions are used to compute the temperature distribution of the tetrahedron closest to the centre of the cube.

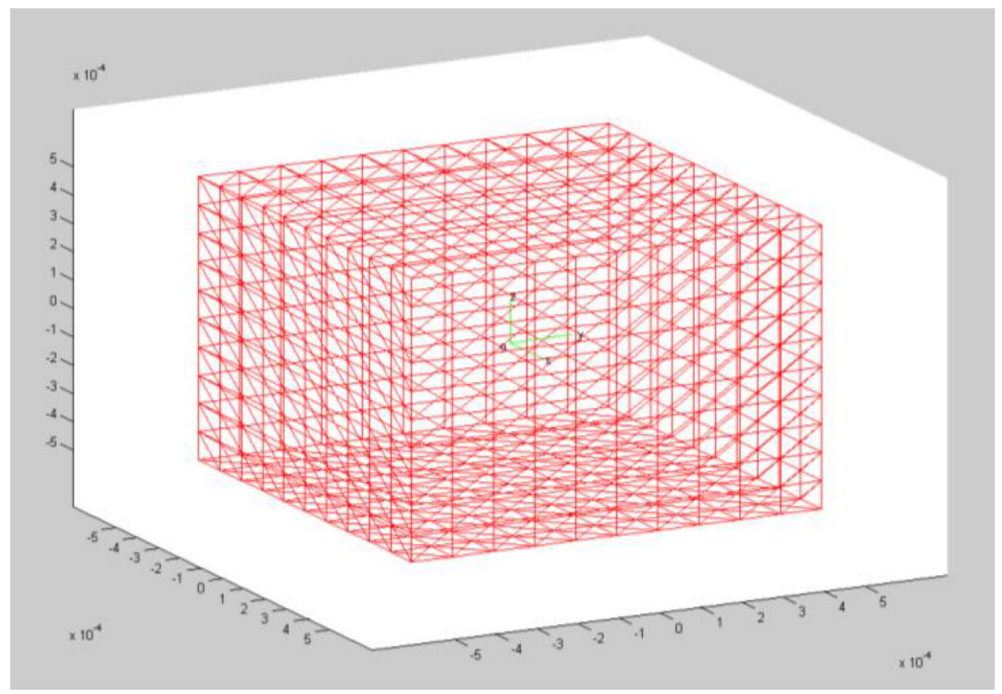

Figure 1: Cube modelled by 1,200 triangles and 3,669 tetrahedrons. 
The TDBEM is compared against Finite Difference ADI [4], Finite Volume method [5] and the following analytical solution from Carslaw \& Jaeger [6].

$$
\begin{gathered}
T=T_{b c}-\frac{64 T_{b c}}{\pi^{3}} \sum_{l=0}^{\infty} \sum_{m=0}^{\infty} \sum_{n=0}^{\infty} \frac{(-1)^{l+m+n}}{(2 l+1)(2 m+1)(2 n+1)} \cos \left(\frac{(2 l+1) \pi x}{2 a}\right) \\
\cdot \cos \left(\frac{(2 m+1) \pi y}{2 b}\right) \cos \left(\frac{(2 n+1) \pi z}{2 c}\right) e^{-\beta t}
\end{gathered}
$$

where

$$
\beta=\frac{\alpha \pi^{2}}{4}\left[\left(\frac{2 l+1}{a}\right)^{2}+\left(\frac{2 m+1}{b}\right)^{2}+\left(\frac{2 n+1}{c}\right)^{2}\right]
$$

and the temperature $T_{b c}$ is the constant boundary condition for the region $-a<x<a$, $-b<y<b,-c<z<c$.

The numerical results are implemented using a time step of $40 \mathrm{~ms}$. The temperature distribution of the tetrahedron the closest to the centre of the cube is plotted in Fig. 2.

It is encouraging to observe that the constant time interpolation TDBEM solution is similar in accuracy to the FVM and Finite Difference ADI method. It can also be observed that the use of stepwise linear functions does not improve the accuracy of the TDBEM solution.

\subsection{Pulsed volumetric heat source internal solution to a rectangular parallelepiped cavity} with a pulse of constant heat generation produced for $t_{1}<t<t_{2}$

Sources are applied throughout the whole volume of the cube from time $t=3 \Delta t$ to $t=13 \Delta t$ with $\Delta t=40 \mathrm{~ms}$ and initial and constant boundary conditions set at $37^{\circ} \mathrm{C}$. Results yielded by the constant time interpolation TDBEM are compared to the finite volume solution, both methods using a similar mesh as the two previous examples, and the finite difference

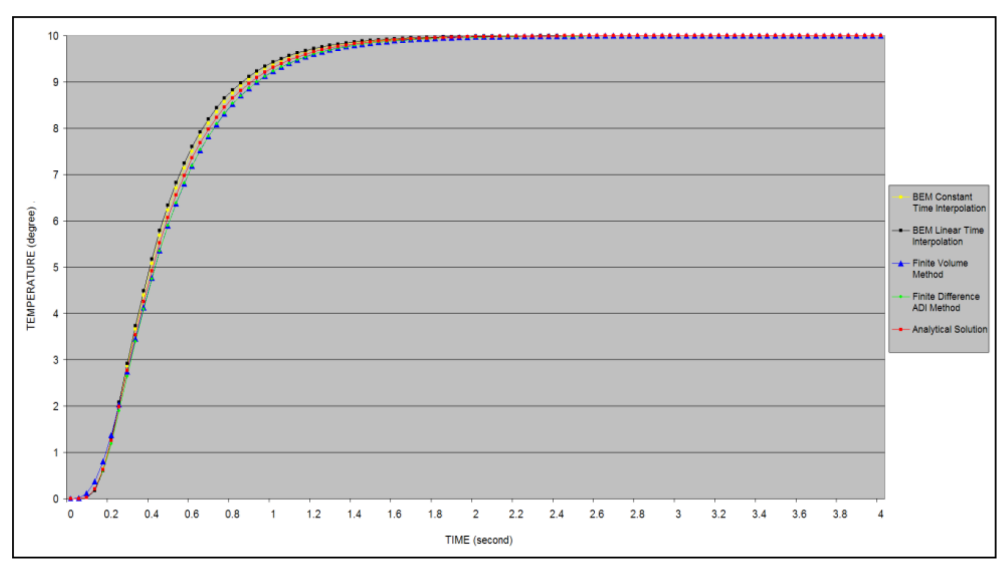

Figure 2: Temperature distribution of the tetrahedron closest to the centre of the cube. Time step used is $40 \mathrm{~ms}$. 


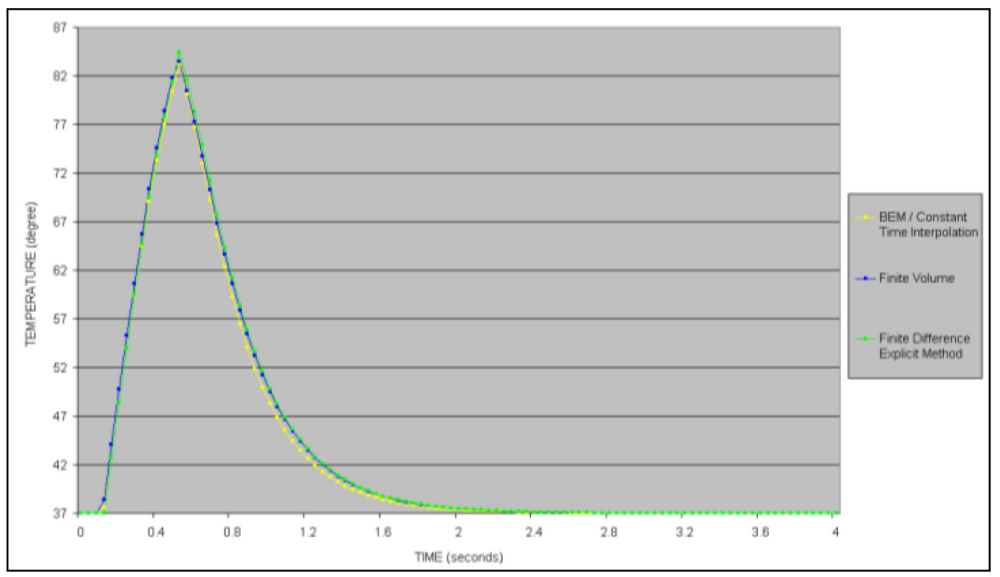

Figure 3: Temperature distribution of the tetrahedron closest to the centre of the cube.

ADI solution generated with 1,771,561 body elements. For a constant heat generation rate $\boldsymbol{A}_{0}=5 \times 10^{8} \boldsymbol{W} . \boldsymbol{m}^{-2}$, Fig. 3 summarises the results and demonstrates better agreement between the TDBEM, FVM and FDM methods with differences less than $3 \%$ at worst.

These verification studies gave us confidence in using the TDBEM for more complex geometries represented by an unstructured mesh.

\section{THE SINGULARITY CHALLENGE}

As mentioned previously, care must be taken in the choice of the time step value. As $\Delta t \rightarrow 0$, the fundamental solutions $T^{*}$ and $q^{*}$ become less and less smooth, their limit being a Dirac delta function as shown in Fig. 4 for the example of $T^{*}$. The independent axis, R, is a measure of the distance between the source and field point in units of the mesh size.

We investigate the accuracy of the TDBEM solution by considering the benchmark problem 3.1 with various time steps, mesh sizes and Gaussian quadrature schemes. The following data are specified:

- Time step 40 ms (Fig. 5).

- Time step 4 ms (Fig. 6).
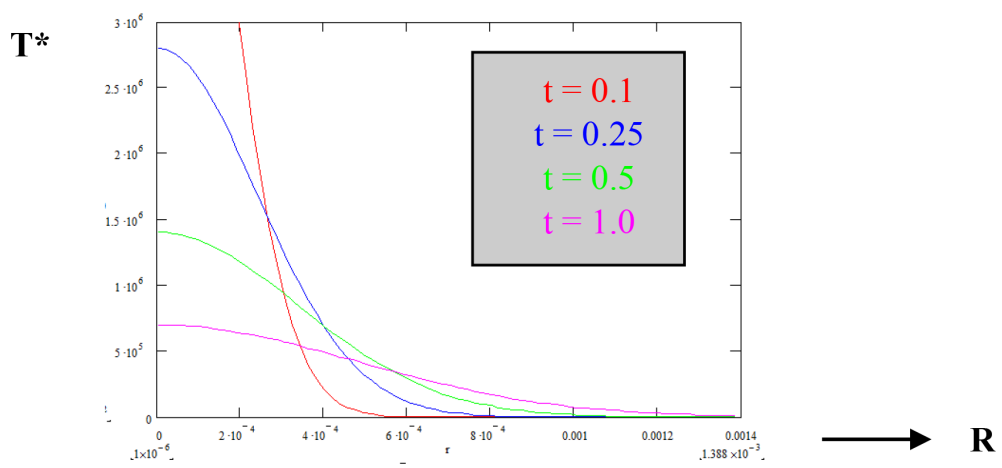

Figure 4: Singularity behaviour of the fundamental solution for decreasing $\Delta \mathrm{t}$. 


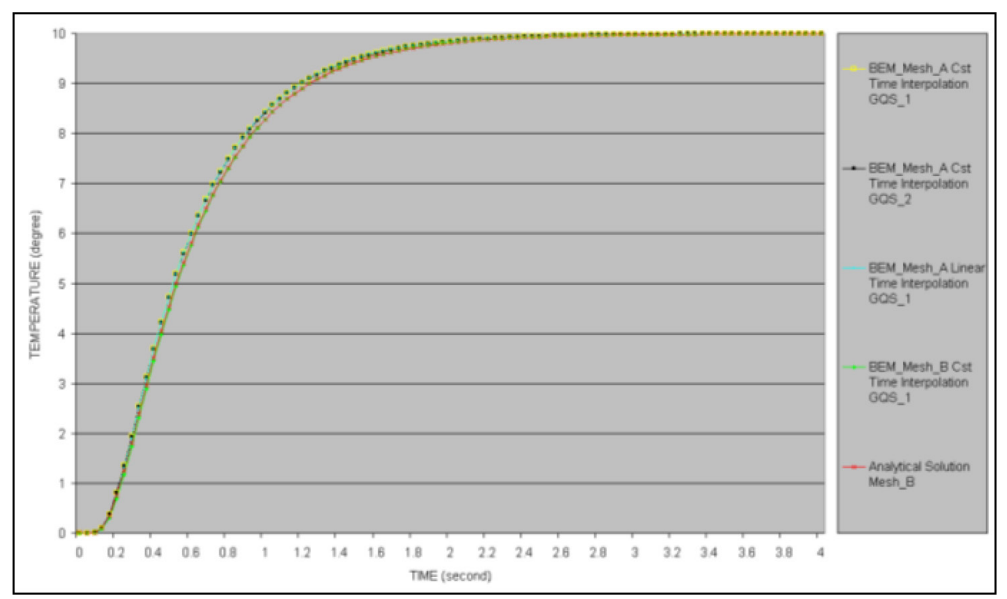

Figure 5: Temperature distribution of the element the closest to the centre of the square cavity, computed using time step $40 \mathrm{~ms}$.

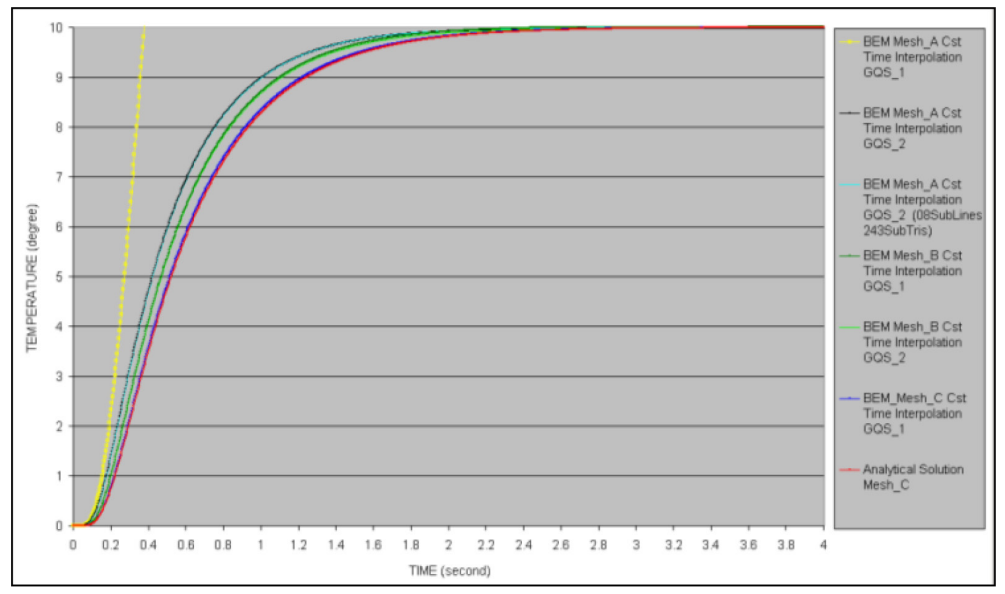

Figure 6: Temperature distribution of the element the closest to the centre of the square cavity, computed using time step $4 \mathrm{~ms}$.

- Gaussian quadrature scheme GQS_1: 12 Gaussian points for boundary elements, 7 Gaussian points for domain elements.

- Gaussian quadrature scheme GQS_2: 48 Gaussian points for boundary elements, 27 Gaussian points for domain elements.

- Mesh A: 40 boundary elements, 200 domain elements.

- Mesh B: 80 boundary elements, 892 domain elements.

- Mesh C: 200 boundary elements, 5,562 domain elements.

Using $\Delta \mathrm{t}=40 \mathrm{~ms}$ and mesh $\mathrm{A}$, it can be deduced from Fig. 5 that the Gaussian quadrature scheme GQS_1 generates a satisfactory numerical integration solution as refinement GQS_2 does not improve the accuracy of the BEM results. The discrepancy observed against the analytical solution is then due to mesh A being too coarse. Thus, a finer mesh B combined 
Table 1: Rules for the space integration of the fundamental solutions $T^{*}$ and $q^{*}$ over a triangle for three dimensional problems and $\Delta \mathrm{t}=40 \mathrm{~ms}$.

\begin{tabular}{ll}
\hline $\mathrm{R}=$ (distance between field and source)/mesh size & GAUSS QUADRATURE \\
\hline$R \geq 2.5$ & 4 \\
$2.5>R \geq 1$ & 7 \\
$1>R \geq 0.3$ & 27 \\
$0.3>R \geq 0.2$ & 27 points/3 sub-triangles \\
$0.2>R \geq 0.08$ & 27 points/9 sub-triangles \\
\hline
\end{tabular}

with GQS_1 offers a satisfactory solution as the results generated get closer to the analytical solution. It can also be seen that the use of stepwise linear functions does not have a significant influence on the results. However, when using the smaller time step $1 \mathrm{~ms}$ (Fig. 6) with mesh A, the first Gaussian quadrature scheme GQS_1 does not prove to be accurate anymore. GQS_2 produces an improvement but the division of the boundary elements into 8 subelements and the division of the domain elements into 243 sub-elements does not improve the solution. With a finer mesh B, GQS_1 and GQS_2 produce similar results. Only a much finer mesh $\mathrm{C}$ allows the BEM to generate results close to the analytical solution.

As emphasised by this example, the relative sizes of geometrical data and time step have to be carefully chosen if accurate results are to be expected. Clearly, there exists a minimum value for $\Delta t$ to ensure accurate integration of the fundamental solution, especially for field points close to the sources. Furthermore, attention should be paid to the fact that the more refined the domain discretisation employed, the closer to the boundary cells integration points become, which also implies the need to refine the boundary integration (or/and discretisation). For the three-dimensional problem considered in this benchmark exercise with a time-step of $40 \mathrm{~ms}$, a series of parametric studies has established a set of rules required to achieve an accuracy of better than $0.05 \%$. Table 1 shows some of these rules for evaluating the $\mathbf{H}$ and $\mathbf{G}$ matrices. Similar rules have been established for the other matrices in both 2D and 3D.

\section{APPLICATION TO OPTICAL MEDICAL DEVICE MODELLING}

Figures 7 and 8 illustrate the 3D case study of an oblique hair follicle target within human skin. The problem consists of approximately 29,000 unstructured tetrahedral elements and 3,000 surface triangular elements.

The skin top surface is uniformly irradiated with a $25 \mathrm{~ms}$ flat-top light pulse and Fig. 9 shows the resultant light fluence for a plane cut through the 3D structure, calculated from a DRBEM computation. Figure 10 shows the temperature-time plots from the TDBEM calculation for the selected elements on the hair follicle indicated in Fig. 8. These temperature curves are used to estimate follicular damage, which occurs when cells are held at temperatures above $42^{\circ} \mathrm{C}$. The temperature calculation uses the DRBEM-derived radiative fluence distribution for the volumetric heat source term in eqn (4).

This case study is interesting in that it demonstrates the necessity of full-parallelisation of the TDBEM for a representative real-world 3D problem, where the problem requires a non-trivial mesh density. Memory requirements were $~ 55$ GB and parallelisation using shared memory threading on a 12 core dual-Xeon architecture reduced a 6 hour serial calculation to just 7 minutes. 


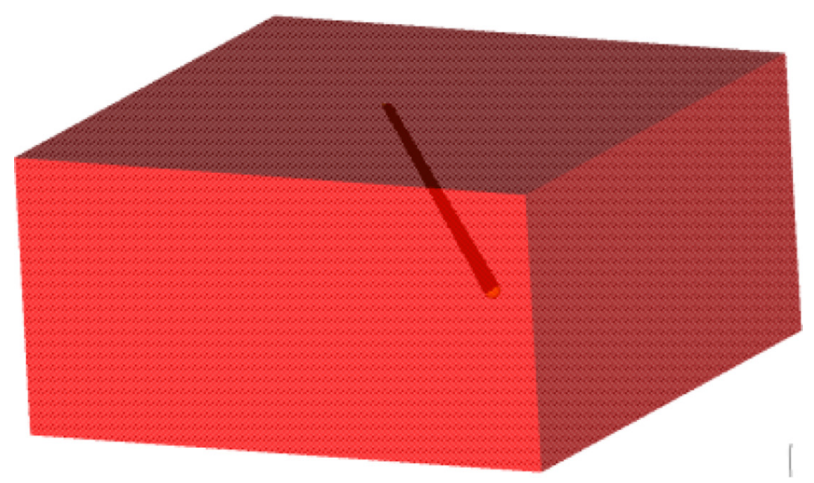

Figure 7: Oblique hair-follicle in tissue.

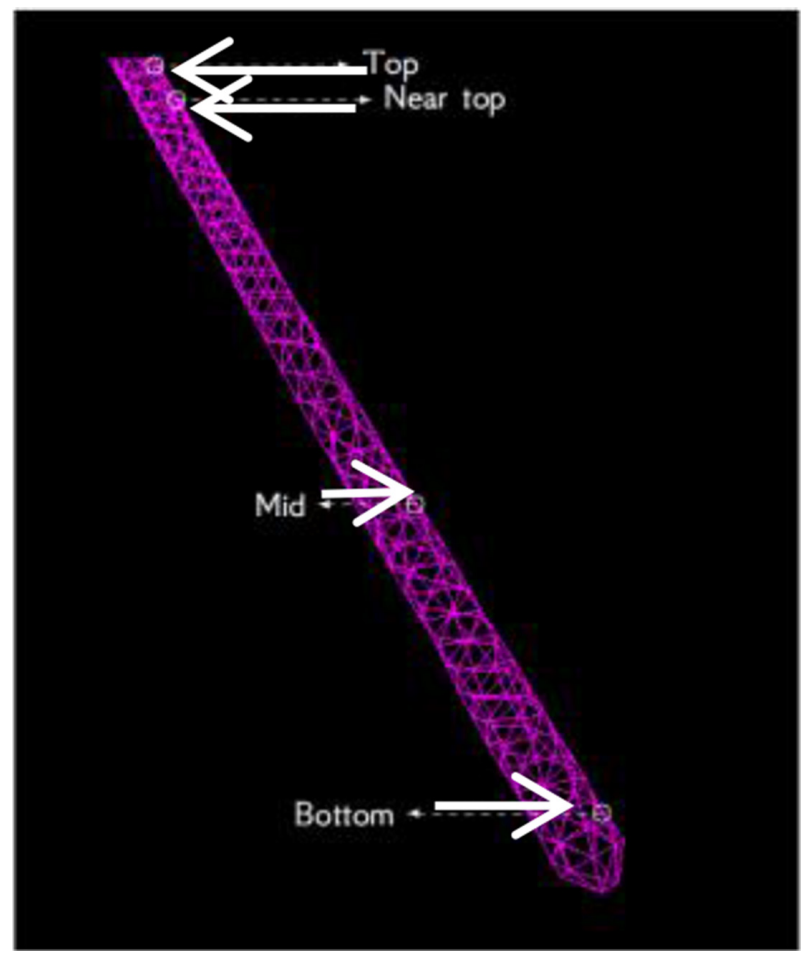

Figure 8: selected points on follicle.

The $\Delta \mathrm{t}$ singularity in the fundamental solution, eqn (2), is explored for this case study. Figure 11 shows the temperature -time plots for three different $\Delta \mathrm{t}$ values for a tetrahedral element at the base of the follicle. Although the peak temperature is very similar in each simulation, the expected asymptotic trend to the tissue temperature of $37^{\circ} \mathrm{C}$ is only evident in the longest $\Delta \mathrm{t}$ value of $25 \mathrm{~ms}$. As Wrobel [1] has previously indicated, this should not be interpreted as a stability problem, but rather as a requirement to use higher quality discretisation and quadrature schemes for the spatial integration when the time-step is reduced. Further systematic study of this accuracy challenge is underway. 


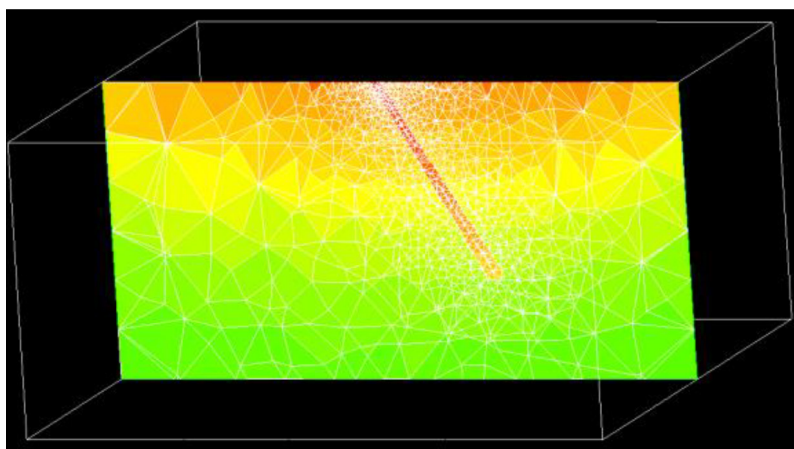

Figure 9: 3D-slice showing radiative fluence obtained from DRBEM used as source terms for temperature calculation.

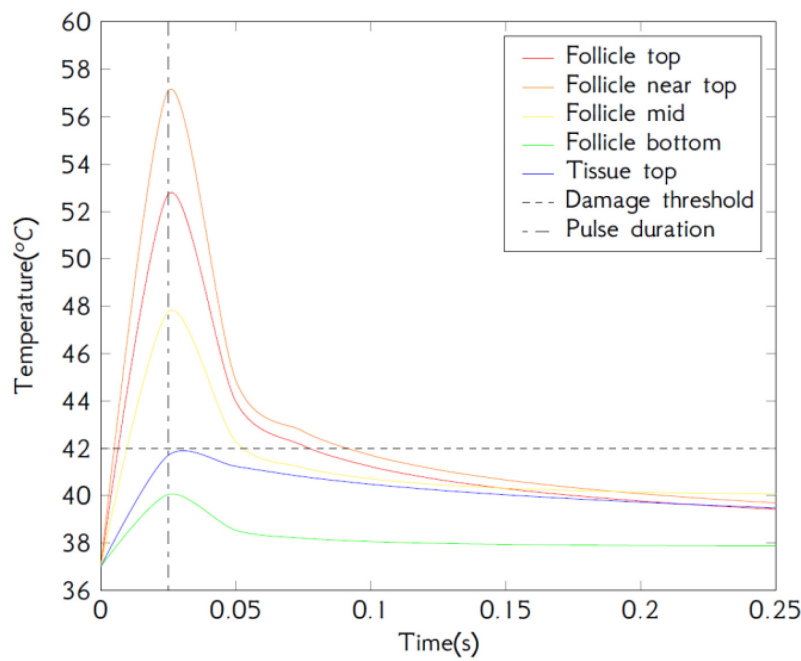

Figure 10: Temperature-time plots for selected points on follicle.

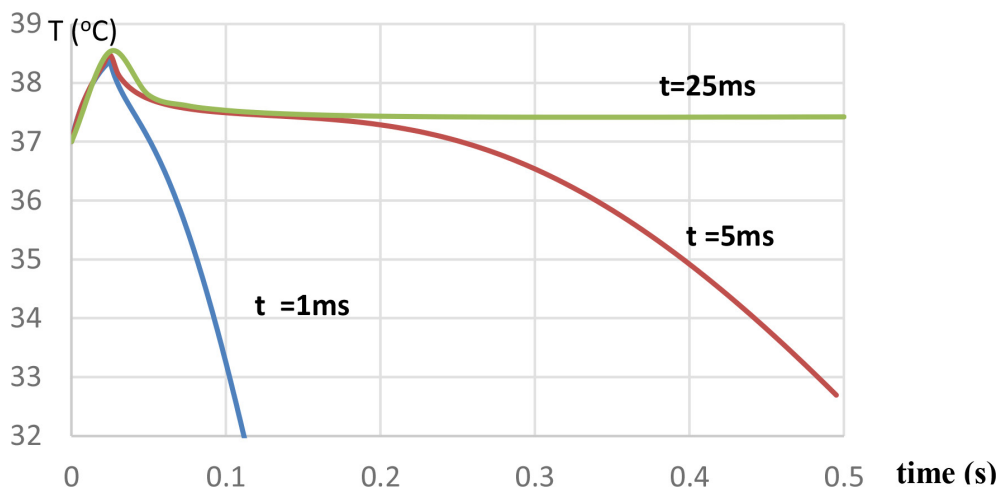

Figure 11: The effect of the singularity on calculated temperature for varying $\Delta \mathrm{t}$. 


\section{CONCLUSIONS}

The TDBEM has been verified against benchmark problems which contain pulsed volumetric heat sources. Subsequent application to a realistic clinical problem indicates that the fully parallelised method can produce fast solutions that permit parametric studies to be undertaken to determine the optimal light pulse characteristics for best therapeutic benefit [7]. Further systematic studies are underway to investigate the accuracy and speed of the TDBEM for combinations of $\Delta t$ and mesh size, and when the number of internal poles, $L$, is reduced, whilst still evaluating the solution at all required $\mathrm{N}_{\mathrm{V}}$ solution points in the domain. Initial comparable studies for the DRBEM used in the radiative calculation suggest that very fast solution times can be obtained while still maintaining acceptable accuracy.

\section{REFERENCES}

[1] Donne, K.E., Marotin, A. \& Al-Hussany, A., Modified dual reciprocity boundary element modeling of collimated light fluence distribution in normal and cancerous prostate tissue during photodynamic therapy. 34th International Conference on Boundary Elements and other Mesh Reduction Methods, Split, Croatia, 2012.

[2] Wrobel, L.C., The Boundary Element Method Vol 1: Applications in Thermofluids and Acoustics, Wiley, 2002.

[3] Brebbia, C.A, Telles, J.C.F. \& Wrobel, L.C., Boundary Element Techniques, SpringerVerlag, 1984.

[4] Daniel, G., Donne, K.E., Song, L., Computer Modelling of Photodynamic Therapy, IPEM Annual Scientific meeting, York, UK, 2004.

[5] MAYA, available at: http://www.mayahtt.com/expertise/thermal

[6] Carslaw, H.S. \& Jaeger, J.C., Conduction of Heat in Solids, 2nd edn., Oxford Clarendon Press, 1959.

[7] Van der Beek, N., Donne, K., Bjerring, P. \& Neumann, M., The Effect of Pulse Train Profile on the Efficacy of PDL Treatments for PWS. Br J Dermatol, July 2015. http://dx.doi.org/10.1111/bjd.14008 